\title{
Role of fecal calprotectin as a biomarker of intestinal inflammation in ulcerative colitis: a prospective study
}

\author{
Danusia Onişor, Alina Boeriu*, Ofelia Pascarenco, Olga Brusnic, \\ Daniela Dobru
}

Departament of Gastroenterology, University of Medicine and Pharmacy Tîrgu Mureş, Romania

\begin{abstract}
Background: The clinical utility of non-invasive markers in the diagnosis and monitoring of ulcerative colitis (UC) has been intensively studied. The aim of our study was to evaluate the value of fecal calprotectin (FC) in differentiating between UC and irritable bowel syndrome (IBS), and in estimating inflammatory activity in UC.

Method: A total number of 140 patients were included in the study. All patients underwent ileocolonoscopy with biopsies, quantitative determination of FC, and blood tests (white blood cell count, CRP, ESR). The severity of UC was assessed by using the Ulcerative Colitis Disease Activity Index (UCDAI) and Mayo endoscopic score.

Results: In patients with active UC the mean values of FC were $373.8+/-146.3 \mu \mathrm{g} / \mathrm{g}$, significantly higher than those in the inactive UC (mean values $36.04+/-13.25 \mu \mathrm{g} / \mathrm{g}$ ), and in IBS $(42.9+/-16.00 \mu \mathrm{g} / \mathrm{g}$ ). In univariate regression analysis, elevated FC levels strongly correlated with pancolitis ( $p=0.0001)$, UCDAI and Mayo scores ( $p=0.0001)$, and elevated CRP levels. In multivariate regression model, FC was positively associated with severe pancolitis, and elevated CRP. The optimal cutoff value of FC for the prediction of severe pancolitis (Mayo score $>$ 3) was $540 \mu \mathrm{g} / \mathrm{g}$. We obtained 71.4\% sensitivity (CI95\%: 41.95-91.6) and 96.1\% specificity (C195\%: 89.2 -99.2) of $F C$ in assessing the severity of inflammation in UC patients.

Conclusion: FC is a promising marker that can be used in clinical practice to select patients with organic intestinal disorders, compared with those with functional disorders. It also correlates very well with the extent of lesions and the severity of clinical symptoms in UC, with increased sensitivity and specificity. .
\end{abstract}

Keywords: fecal calprotectin, ulcerative colitis, irritable bowel syndrome

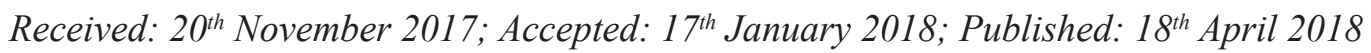

\section{Background}

Ulcerative colitis (UC) is a chronic disorder characterized by mucosal inflammation at the level of the colon and rectum, with a typically relapsing and remitting course [1]. Endoscopic mucosal healing represents the main therapeu- tic target, leading to sustained clinical remission and reduced hospitalization rates and need for surgery [2].

The current gold standard in the assessment of intestinal inflammation in UC is colonoscopy with mucosal biopsies. Unfortunately, the

\footnotetext{
*Corresponding author: Alina Boeriu, University of Medicine and Pharmacy Tirgu Mures Tirgu Mures, Mures, Romania. E-mail: aboeriu@gmail.com
} 
endoscopic procedure is invasive and requires an uncomfortable preparation. Colonoscopy in patients with active UC may be associated with serious complications such as perforation and bleeding, especially in the case of severe lesions. Therefore, noninvasive methods are required to estimate the severity and the extension of the disease [1].

Irritable bowel syndrome (IBS) is a prevalent symptom-based disorder, characterized by the association of abdominal pain with altered bowel habits [3]. In clinical practice, differential diagnosis between IBS and IBD is mainly based on endoscopic and histological evaluation. However, recent studies have focused on the analysis of the clinical utility of biomarkers, as alternative non-invasive methods for differentiating IBD and IBS patients. The value of surrogate markers for intestinal inflammation, such as C-reactive protein (CRP), erythrocyte sedimentation rate (ESR), fecal calprotectin (FC), and fecal lactoferrin, has been considered in clinical research [3].

CRP is produced by the liver in response to proinflammatory cytokines [3]. Lactoferrin, a globular glycoprotein, is present in secondary granules of polymorphonuclear neutrophils. Calprotectin is a zinc and calcium binding protein, released into the intestinal lumen by activation of leukocytes or as a result of their degradation. This protein can resist metabolic degradation caused by intestinal bacteria and it is stable in feces up to one week at room temperature [4]. The value of both fecal lactoferrin and fecal calprotectin for the assessment of inflammatory activity in UC and Crohn's disease was proved in various clinical studies [5]. Based on published data, FC was progressively used as a surrogate marker for mucosal inflammation in patients with UC [6].

Our study aimed to evaluate the clinical utility of fecal calprotectin (FC) in differentiating between active UC and IBS and in the assessment of inflammatory activity in UC.

\section{Material and Method}

This study was carried out at the Department of Gastroenterology, County Hospital, Tîrgu Mureş. A total number of 140 patients with UC or IBS were enrolled during one year, from September 2016 to September 2017. The study was approved by the Ethics Committee of our Institution. All the patients agreed to participate and were provided a written informed consent for all procedures (collection of blood and stool samples, endoscopic procedures). Exclusion criteria were Crohn's disease, recent treatment with NSAIDs, Aspirin, antibiotics, infectious gastroenterocolitis (including pseudomembranous colitis), pregnancy, microscopic colitis, and history of digestive tract neoplasia.

A survey was conducted to identify the differences between FC levels and blood tests in three distinct groups of patients: patients with active UC (group Ia) compared with patients with UC in remission (group Ib); patients with UC (group I) compared with patients with IBS (group II).

A total number of 100 patients were diagnosed with UC (group I) based on clinical, endoscopic, and histological criteria. Indications to perform ileocolonoscopy in these patients were clinically active signs (diarrhea, abdominal pain, or rectal bleeding), evaluation of endoscopic disease activity during the treatment, or dysplasia surveillance in long-standing UC. The severity of UC was assessed by using the Ulcerative Colitis Disease Activity Index (UCDAI) (mild< 3 , moderate between $3-7$, and severe $>7$ ). The Mayo endoscopic score (0-normal mucosa, 1-erythema, 2-erosion, 3-ulcer) was used for the assessment of endoscopic activity.

Initially, 92 patients presented active disease and were included in group Ia, while eight patients with long-standing UC were in clinical and endoscopic remission and were included in group Ib. On further evaluation, 39 patients in- 
cluded in group Ia achieved clinical (no diarrhea, abdominal pain, or rectal bleeding) and endoscopic remission (endoscopic mucosal healing) after treatment, and were included in group $\mathrm{Ib}$. Thus, group Ib comprised a total number of 47 patients who achieved clinical and endoscopic remission: 8 patients in remission on initial presentation, and 39 patients who achieved remission on further evaluation.

Group II included 40 patients with IBS. The diagnosis of IBS was established according to ROME IV criteria. Ileocolonoscopy with mucosal biopsy was performed to exclude other organic diseases.

Stool samples were collected to determine FC by using the BÜHLMANN Quantum Blue ${ }^{\circledR}$ FC test. The test is designed for the selective measurement of calprotectin antigen by sandwich immunoassay. Blood samples were collected for CRP, white blood cell count, and ESR determination. The cut-off values were: FC $\geq 50 \mu \mathrm{g} / \mathrm{g}, \mathrm{CRP}$ $\leq 0.5 \mathrm{mg} / 1$, and ESR under $10 \mathrm{~mm} / \mathrm{h}$.

Laboratory tests were performed on initial presentation for all patients (100 UC patients, 40 IBS patients). Additionally, subsequent laboratory investigations were performed only in those patients with active UC who achieved clinical and endoscopic remission after the treatment (39 patients included in group Ib). Correlations were made between FC levels, CRP, white blood cell count, and ESR in patients with active disease (group Ia), compared with patients in remission (group Ib), and patients with IBS (group II) respectively.

\section{Statistical analysis}

Qualitative data were presented as amounts and percentages. The association between qualitative variables was assessed using the Chisquare test or the Fisher exact test. Quantitative data were presented as mean and standard deviation (for normal distribution). To check for differences between two independent groups of quantitative data, the Student's T-test for independent samples (for normal distribution) and Mann Whitney test (for nongaussian distribution) were used. We used the Pearson correlation coefficient. The independent variables were: pancolitis, left-sided colitis, extensive colitis, proctosigmoiditis, age, and sex. The relationship between FC and independent variables was assessed with binomial logistic regression as appropriate statistical methods. In the development of the regression multivariate model, we selected all variables with a p-value less than 0.05 in univariate regression analysis along with all variables of known clinical importance. Results were presented as coefficients and 95\% CI. For all statistical tests the significance level alpha was set at 0.05 , and the two-tailed p-value was calculated. We used the SPSS statistical software package 22.0 (SPSS, Inc., Chicago, IL, USA) for all statistical analyses.

\section{Results}

We applied an ANOVA test $(p=0.0001)$, and the Bonferroni Multiple Comparison Test to compare the differences between the three samples. We noticed that the mean values of FC-Ia were $373.8+/-146.3 \mu \mathrm{g} / \mathrm{g}$, significantly higher compared to FC-Ib $(36.04+/-13.25 \mu \mathrm{g} / \mathrm{g})$, and compared to FC-II $(42.9+/-16.00 \mu \mathrm{g} / \mathrm{g})$. No statistically significant difference was detected between FC-Ib and FC-II (Fig. 1). When we compared the mean values of FC in patients with active UC (FC-Ia) with the mean values of FC in patients without endoscopic lesions (FC$\mathrm{Ib}$ and FC-II), we obtained significantly higher values in the first group $(373.8+/-146.3$ versus $39.15+/-14.88 \mu \mathrm{g} / \mathrm{g}$ ).

Group Ia, with active UC, comprised 92 patients, with a mean age of 41.1 years (18 -74 years). Within this group, FC values were as- 


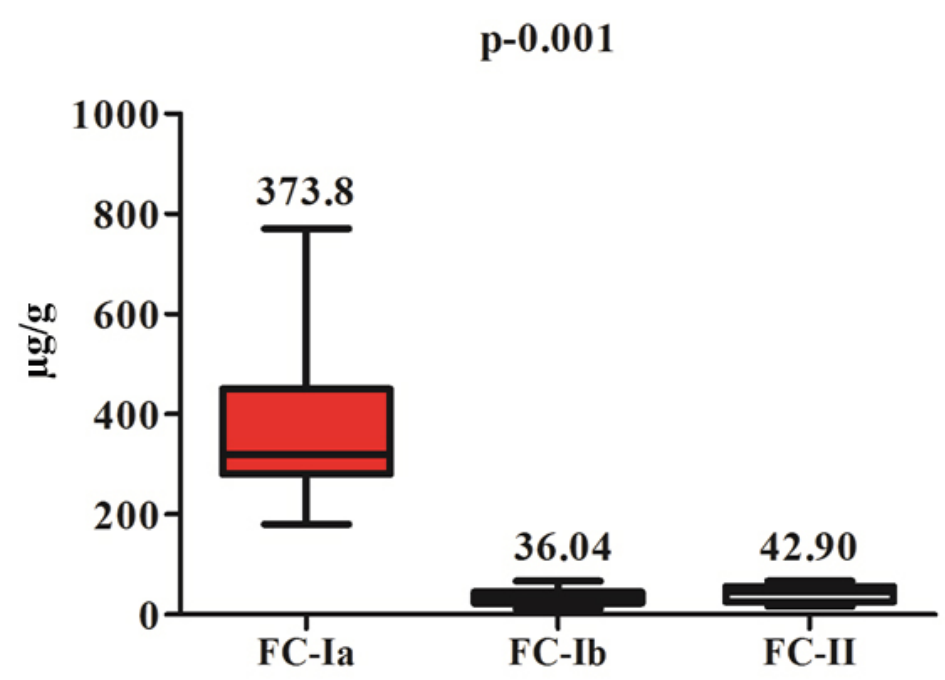

Fig.1. Inferential statistics of calprotectin values for the three study groups

sessed by gender, disease extension (proctosigmoiditis, left-sided colitis, extensive colitis, pancolitis) and severity scores (UCDAI and Mayo score). We observed no significant alteration of FC in the two different genders of our study population. Significantly higher amounts of FC were detected in patients with active proc- tosigmoiditis and left-sided colitis, and also in patients with pancolitis. A statistically significant increasing of $\mathrm{FC}$ values with the increasing of UC disease severity (UCDAI $>3$, and Mayo endoscopic score $>2$ ) was associated (Table I).

By applying the Pearson correlation test, FCIa correlates positively and highly significantly

Table I. Comparative mean values of FC $(\mu \mathrm{g} / \mathrm{g})$ by disease extension and severity scores

\begin{tabular}{|c|c|c|c|c|c|}
\hline Variables & & $\mathbf{N}$ & Mean & Std. Deviation & P value \\
\hline \multirow{2}{*}{ Sex* } & $\mathrm{F}$ & 56 & 370.96 & 148.630 & \multirow{2}{*}{0.81} \\
\hline & $\mathrm{M}$ & 36 & 378.33 & 144.578 & \\
\hline \multirow{2}{*}{ Proctosigmoidits* } & Yes & 18 & 256.29 & 46.175 & \multirow{2}{*}{0.001} \\
\hline & No & 74 & 400.49 & 148.210 & \\
\hline \multirow{2}{*}{ Left-sided colitis* } & Yes & 31 & 321.77 & 90.471 & \multirow{2}{*}{0.004} \\
\hline & No & 61 & 399.05 & 161.431 & \\
\hline \multirow{2}{*}{ Extensive colitis* } & Yes & 28 & 397.79 & 90.751 & \multirow{2}{*}{0.30} \\
\hline & No & 64 & 363.38 & 164.382 & \\
\hline \multirow{2}{*}{ Pancolitis* } & Yes & 15 & 601.14 & 168.595 & \multirow{2}{*}{0.001} \\
\hline & No & 77 & 333.05 & 97.044 & \\
\hline \multirow[b]{3}{*}{ UCDAI** } & $<3$ & 27 & 266.22 & 63.370 & 0.01 \\
\hline & $3-7$ & 42 & 367.14 & 93.900 & 0.0001 \\
\hline & $>7$ & 23 & 512.43 & 181.594 & 0.002 \\
\hline \multirow[b]{3}{*}{ MAYO** } & 1 & 26 & 262.46 & 58.761 & 0,01 \\
\hline & 2 & 39 & 362.28 & 93.231 & 0.0001 \\
\hline & 3 & 27 & 497.81 & 174.139 & 0.003 \\
\hline
\end{tabular}

Note. Data were expressed by mean $+/-\mathrm{SD}, *$ Student test, $* *$ - Anova test + Bonferroni multi-comparability test 
with leukocytes levels ( $\mathrm{p}=0.0001, \mathrm{r}=0.624)$, CRP $(p=0.0001, r=0.732)$, and ESR $(p=0.0001$, $\mathrm{r}=0.704)$. The increase of these markers is associated with elevated levels of FC.

In the univariate regression analysis, we analyzed the relationship between FC-Ia as the dependent variable, and some independent variables (age, disease extension, severity, leucocyte level, CRP, and ESR). Elevated FC-Ia levels are influenced by the presence of pancolitis $(p=0.0001)$, and correlate with high UCDAI and Mayo scores $(\mathrm{p}=0.0001)$, elevated CRP levels $(p=0.0001)$, and elevated ESR $(p=0.0001)$. An increased white blood cell count between $9.0-15.0 \times 10^{9} / \mathrm{L}$ is positively associated with increased FC levels (Table II).
Those variables that in univariate regression analysis were statistically significant when associated with FC were introduced to a multivariate regression model. We found that FC is positively associated with severe pancolitis, and elevated CRP (Table III).

The optimal cutoff value of FC for the prediction of severe pancolitis (Mayo score $>3$ ) was $540 \mu \mathrm{g} / \mathrm{g}$. The area under the receiver operating characteristic curve (AUC) was 0.903 . The performance values of FC while assessing the severity of inflammation in patients with UC were: 71.4\% sensitivity (CI95\%: 41.95-91.6), 96.1\% specificity (CI95\%: 89.2-99.2), 76.9\% PPV (CI95\%: 46.2-95.0), and 94.9\% NPV (CI 95\%: 87.5-98.6).

Table II. Univariate regression for considered independent variable

\begin{tabular}{lcccccc}
\hline \multirow{2}{*}{$\begin{array}{l}\text { Variables } \\
\text { B }\end{array}$} & \multirow{2}{*}{ S.E. } & \multirow{2}{*}{ Beta } & \multirow{2}{*}{ P value } & \multicolumn{2}{c}{$\begin{array}{c}\text { 95\% Confidence Interval } \\
\text { for B }\end{array}$} \\
\cline { 5 - 7 } & & & & & Lower & Upper \\
\hline Age & -1.653 & 1.160 & -0.149 & 0.158 & -3.959 & 0.652 \\
\hline Proctosigmoiditis & -144.199 & 36.477 & -0.385 & 0.0001 & -216.667 & -71.731 \\
\hline Left-sided colitis & -77.282 & 31.688 & -0.249 & 0.017 & -140.235 & -14329 \\
\hline Extensive colitis & 34.411 & 33.135 & 0.109 & 0.01 & -31.418 & 100.239 \\
\hline Pancolitis & 268.092 & 32.011 & 0.662 & 0.0001 & 204.495 & 331.688 \\
\hline UDCAI & 122.293 & 16.376 & 0.619 & 0.0001 & 89.759 & 154.827 \\
\hline MAYO & 117.819 & 15.943 & 0.615 & 0.0001 & 86.146 & 149.493 \\
\hline Leucocytes x10/L & 0.026 & 0.003 & 0.624 & 0.0001 & 0.019 & 0.033 \\
\hline CRP mg/L & 38.934 & 3.814 & 0.732 & 0.0001 & 31.356 & 46.512 \\
\hline ERS mm/h & 5.992 & 0.638 & 0.704 & 0.0001 & 4.725 & 7.250 \\
\hline
\end{tabular}

Tabel III. Multivariate regression model table

\begin{tabular}{lccccccc}
\hline \multirow{2}{*}{ Model } & \multicolumn{3}{c}{ Unstandardized Coefficients } & $\begin{array}{c}\text { Standardized Co- } \\
\text { efficients }\end{array}$ & & \multicolumn{2}{c}{$\begin{array}{c}\text { 95\% Confidence Interval } \\
\text { for B }\end{array}$} \\
\cline { 2 - 3 } & $\mathrm{B}$ & Std. Error & Beta & & & $\begin{array}{c}\text { Lower } \\
\text { Bound }\end{array}$ & Upper Bound \\
\hline Left-sided colitis & -21.796 & 24.298 & -0.070 & 0.372 & -70.125 & 26.532 \\
\hline Pancolitis & 99.853 & 35.622 & 0.246 & 0.006 & 29.003 & 170.704 \\
\hline UDCAI & 20.557 & 20.859 & 0.104 & 0.327 & -20.929 & 62.044 \\
\hline MAYO & 12.722 & 20.437 & 0.066 & 0.005 & -27.925 & 53.370 \\
\hline Leucocytes & 0.002 & 0.004 & 0.055 & 0.565 & -0.006 & 0.010 \\
\hline CRP & 14.922 & 4.921 & 0.281 & 0.003 & 5.136 & 24.707 \\
\hline ESR & 2.607 & 0.746 & 0.306 & 0.01 & 1.123 & 4.092 \\
\hline
\end{tabular}




\section{Discussion}

The differential diagnosis of an organic bowel disease compared to IBS can be challenging. The development of an accurate and non-invasive test, able to distinguish organic from functional disorders, becomes a major goal in clinical practice.

In our analysis, we focused on patients with $\mathrm{UC}$, which represent the majority of IBD patients in our geographical area. A recent study published by Romanian authors regarding the diagnostic delay in Romanian patients with IBD reported the higher incidence of UC compared with Crohn's disease in Transylvania (41.5\% $\mathrm{UC}, 13.6 \% \mathrm{CD}$ ), and $55.6 \%$ indeterminate colitis [7]. In our daily practice, UC and IBS represent frequent disorders, with similar symptoms, such as abdominal pain, bloating, and diarrhea. Rectal bleeding is a red flag symptom, indicating the need for endoscopic examination. Even so, the diagnostic delay in IBD patients represents the reality in practice and may be explained by the misinterpretation of symptoms in case of IBS. Rectal bleeding may also be considered to be caused by a benign perianal disease. [7] In this situation, it will be helpful to have a tool that could alert us for the severity of the disease, especially in those patients without rectal bleeding, to prioritize them for detailed structural examinations (colonoscopy with biopsies). Thus, after the evaluation of symptoms and clinical signs, inflammatory marker dosing should be included in the differential diagnosis algorithm between IBS and IBD [8].

The usefulness of surrogate markers such as fecal lactoferrin, FC, CRP, and ESR were evaluated in numerous clinical studies, which included patients suffering from UC, Crohn's disease, and IBS. In a recent meta-analysis, Menees et al. [9] showed that low levels of CRP (below 0.5 $\mathrm{mg} / \mathrm{dl}$ ) and FC (below $40 \mu \mathrm{g} / \mathrm{g}$ ) could exclude IBD in patients with IBS. CRP and FC may be used to select those patients at higher risk for organic disease, who should undergo further structural evaluation.

Initial studies of FC focused on its ability to differentiate between IBD and IBS [10]. Tibble et al. [11] in a survey comprising 602 patients reported $89 \%$ sensitivity and $79 \%$ specificity of FC for the detection of organic bowel disease. By combining FC with ROME questionnaire, a predictive value for IBS approaching 100\% was reported $[10,11]$.

In a recent study, Kotze et al. [12] showed that FC helps to differentiate active and inactive IBD patients, and also to distinguish between IBS and IBD. Other studies also proved that FC could differentiate between active and inactive IBD [5]. Xiang JY et al. [4] reported that FC concentrations are significantly higher in patients with active UC than in patients with inactive disease. Besides, FC levels are higher in patients with inactive UC than in controls. These results were confirmed by Elsaadany et al. [13] who demonstrated a highly significant increase of FC levels in patients with active UC, compared with patients with inactive UC and IBS.

Similarly, our study proved the statistically significant differences between FC levels in the studied samples. In patients with active UC the mean values of FC were $373.8+/-146.3 \mu \mathrm{g} / \mathrm{g}$, significantly higher than in case of inactive UC (mean values $36.04+/-13.25 \mu \mathrm{g} / \mathrm{g}$ ), and in IBS $(42.9+/-16.00 \mu \mathrm{g} / \mathrm{g})$. Since significantly higher levels of FC were determined in patients with active disease compared with patients in remission, we can conclude that a proper estimation of a disease can be achieved by measuring FC for the optimization of early therapy.

In agreement with previously published data, we obtained significantly higher FC levels $(p<0.05)$ in patients with endoscopic lesions (Ia) compared with patients without endoscopic lesions (Ib), and with IBS (II). 
FC represents a highly sensitive and specific marker of mucosal inflammation $[14,15]$. We obtained $71.4 \%$ sensitivity, $96.1 \%$ specificity, $76.9 \% \mathrm{PPV}$, and $94.9 \% \mathrm{NPV}$ of FC in the assessment of the severity of inflammation in UC patients. In the meta-analyses performed by von Roon et al. [16], and van Rheenan et al. [17], the authors reported FC sensitivity and specificity for IBD above $90 \%$.

In univariate analysis, we obtained a statistically significant association between FC levels and UCDAI score and the Mayo score, leukocyte values, CRP, and ESR. In the multivariate regression analysis of FC with all variables mentioned above, a strong correlation was obtained with pancolitis, Mayo endoscopic score, and CRP. Our findings are in agreement with previously published data, which showed a strong correlation of FC with other markers of UC activity (UCDAI, ESR, CRP, leukocyte levels) [14].

In our study, both FC and CRP were significantly correlated with extensive severe pancolitis, findings consistent with previously published reports. FC levels proved to be significantly linked to endoscopic UC activity, which was assessed using the Mayo score [18].

The correlation between the extent of the disease in UC and FC levels still represents a debatable subject. In our study, FC did not consistently correlate with the extension of endoscopic lesions. Thus, in univariate analysis, left-sided colitis and pancolitis were statistically significant and could be associated with higher FC levels, while proctosigmoiditis and extensive colitis were not statistically significant and could not be associated with increased FC levels.

Previous studies confirmed a lower correlation between FC level and the location of inflammation in the gastrointestinal tract [18]. In fact, only the intensity of mucosal inflammation is linked to elevated FC levels. Ricanek et al. found similar FC levels in both left-sided colitis and pancolitis, with significantly lower levels of proctitis [19].

The discrepancy between the results obtained in various studies could be explained by the different modalities used to assess the extension and severity of inflammation in UC. A modified Mayo endoscopic score, based on the analysis of Mayo scores for each of the five colonic segments, was proposed by Lobaton et al. [20]. Kawashima et al. [1] recently demonstrated that FC level correlates with the severity and the extension of lesions in UC. The authors used the maximum endoscopic score, and also the sum of Mayo scores for all colonic segments to estimate the endoscopic severity of the disease [21].

In other published reports, the endoscopic score was established as the maximum score obtained on the endoscopic examination of the colon and rectum [22-24]. In our study, we used the same modality to determine the endoscopic score, by examining the segment of the colon and rectum with the most severe endoscopic lesions. This may lead to the lack of correlation of FC level with the extension of the disease (FC was not statistically significant and did not correlate with extensive colitis). However, the strong association between FC and endoscopic severity in the most severely affected part of the colon was proved in our study, as well as in previously published reports [21-23]. A cutoff value of $540 \mu \mathrm{g} / \mathrm{g}$ has been demonstrated to be predictive for pancolitis with severe endoscopic lesions (Mayo 3).

Although FC is a very sensitive marker for the inflammation at the level of the gastrointestinal tract as previously discussed, it is also non-specific, since increased levels are also detected in colorectal cancer, infections, polyps, and enteropathy caused by nonsteroidal anti-inflammatory drugs NSAIDs [8]. Its use in primary care is limited, but it is helpful in reducing the number of unnecessary referrals of patients with IBS for specialist investigations (including unnecessary colonoscopies) [25]. According to the 
manufacturer, patients with FC levels below 50 $\mu \mathrm{g} / \mathrm{g}$ do not present inflammation at the level of the gastrointestinal tract and most likely do not need invasive procedures. FC values between 50 and $200 \mu \mathrm{g} / \mathrm{g}$ can indicate inflammation caused by NSAIDs, mild diverticulitis, or IBD in remission phase. Repeating the assessment and additional investigations are suggested in these patients. FC values above $200 \mu \mathrm{g} / \mathrm{g}$ indicate active inflammation in the gastrointestinal tract. Further research is required to assess the optimal cut-off values of FC, and repeat testing strategies in patients with intermediate levels of FC [25].

\section{Conclusions}

Fecal calprotectin is a reliable non-invasive marker of intestinal inflammation. The detection of raised FC levels should signal an organic disease such as IBD and the necessity of performing additional invasive investigations. Determining FC level proved its utility in the differential diagnosis algorithm between IBS and ulcerative colitis.

Calprotectin and CRP represent reliable indicators of the severity of the disorder in UC. FC dosing can be used in routine clinical practice to monitor the activity of the disease and treatment efficacy. An elevated FC level can indicate a possible recurrence of lesions in patients in clinical remission and can predict relapse. FC is recommended as part of the diagnostic investigation that helps clinicians to avoid diagnostic delay, to follow-up patients with ulcerative colitis, and to adjust therapy.

\section{Conflict of interest}

The authors declare no conflict of interest.

\section{References}

1. Kousaku K, Shunji I, Takafumi Y, Nobuhiko F, Naoki O, Hideaki K, et al. Fecal calprotectin level correlat- ed with both endoscopic severity and disease extent in ulcerative colitis. BMC Gastroenterology. 2016 Apr; 16:47. DOI: 10.1186/s12876-016-0462-z

2. Meucci G, Fasoli R, Saibeni S, Valpiani D, Gullotta R, Colombo E, et al. IG-IBD. Prognostic significance of endoscopic remission in patients with active ulcerative colitis treated with oral and topical mesalazine: a prospective, multicenter study. Inflamm Bowel Dis. 2012 Jun;18(6):1006-10. doi: 10.1002/ibd.21838. Epub 2011 Aug 9. DOI: 10.1002/ibd.21838

3. Longstreth GF, Thomson WG, Chey WD, Houghton LA, Mearin F, Spiller RC. Functional bowel disorders. Gastroenterology. 2006 Apr;130(5):1480-91. DOI: 10.1053/j.gastro.2005.11.061

4. Xiang JY, Ouyang Q, Li GD, Xiao NP. Clinical value of fecal calprotectin in determining disease activity of ulcerative colitis. World J Gastroenterol. 2008 Jan;14(1): 53-7. DOI: $10.3748 /$ wjg. 14.53

5. Assadsangabi A, Lobo AJ. Diagnosing and managing inflammatory bowel disease. Practitioner. 2013 JulAug;257(1763):13-8.

6. Daperno M, Castiglione F, de Ridder L, Dotan I, Färkkilä M, Florholmen J, et al. Scientific Committee of the European Crohn's and Colitis Organization. Results of the 2nd part Scientific Workshop of the ECCO. II: Measures and markers of prediction to achieve, detect, and monitor intestinal healing in inflammatory bowel disease. J Crohns Colitis. 2011 Oct;5(5):484-98. DOI: 10.1016/j.crohns.2011.07.003

7. Zaharie R, Tantau A, Zaharie F, Tantau M, Gheorghe $\mathrm{L}$, Gheorghe $\mathrm{C}$, et al. Diagnostic delay in roumanian patients with inflammatory bowel disease: risk factors and impact on the disease course and need for surgery. $\mathrm{J}$ Crohns Colitis. 2016 Mar;10(3):306-14. DOI: 10.1093/ ecco-jcc/jjv215

8. D'Angelo F, Felley Christian, Frossard JL. Calprotectin in daily practice: where do we stand in 2017. Digestion. 2017;95(4):293-301. DOI: 10.1159/000476062

9. Menees SB, Powell C, Kurlander J, Goel A, Chey WD. A Meta-Analysis of the Utility of C-Reactive Protein, Erythrocyte Sedimentation Rate, Fecal Calprotectin, and Fecal Lactoferrin to Exclude Inflammatory Bowel Disease in Adults With IBS. Am J Gastroenterol. 2015 Mar;110(3):444-54. DOI: 10.1038/ajg.2015.6

10. Walsham NE, Sherwood RA. Fecal calprotectin in inflammatory bowel disease. Clin Exp Gastroenterol. 2016 Jan; 9:21-29.

11. Tibble JA, Sigthorsson G, Foster R, Forgacs I, Bja- 
rnason I. Use of surrogate markers of inflammation and ROMA criteria to distinguish organic from nonorganic intestinal disease. Gastroenterology. 2002 Aug;123(2):450-60. DOI: 10.1053/gast.2002.34755

12. Kotze LM, Nisihara RM, Marion SB, Cavassani MF, Kotze PG. Fecal calprotectin: levels for the ethiological diagnosis in Brazilian patients with gastrointestinal symptoms. Arq Gastroenterol. 2015 Jan-Mar;52(1):50-4. DOI: 10.1590/S0004-28032015000100011

13. Elsaadany HM, Almaghraby MF, Edrees AA, Elsherbiny YM, Kumaet RK. Utility of fecal calprotectin as a discriminative biomarker between ulcerative colitis and irritable bowel syndrome and its ability to be used for the assessment of the remission stage of ulcerative colitis. Egypt J Intern Med. 2016 May; 28:21-27. DOI: $10.4103 / 1110-7782.182956$

14. Nouh MAE, Ali AAE, El Halim EFA, Mohamed HI, El Ghany AMA, Badawy AM. Calprotectin as a fecal marker for diagnosis and follow-up in patients with ulcerative colitis. Menoufia Med J. 2014 May; 27:35-43. DOI: $10.4103 / 1110-2098.132726$

15. Vieira A, Fang CB, Rolim EG, Klug WA, Steinwurz F, Rossini LG, et al. Inflammatory bowel disease activity assessed by fecal calprotectin and lactoferrin: correlation with laboratory parameters, clinical, endoscopic and histological indexes. BMC Res Notes. 2009 Oct 29;2:221. DOI: 10.1186/1756-0500-2-221

16. von Roon AC, Karamountzos L, Purkayastha S, Reese GE, Darzi AW, Teare JP, et al. Diagnostic precision of fecal calprotectin for inflammatory bowel disease and colorectal malignancy. Am J Gastroenterol. 2007 Apr;102(4):80. DOI: 10.1111/j.15720241.2007.01126.x

17. van Rheenen PF, E Van de Vijver, V Fidler. Faecal calprotectin for screening of patients with suspected inflammatory bowel disease: diagnostic meta-analysis. BMJ. 2010 Jul;341:c3369. DOI: 10.1136/bmj.c3369

18. Moniuszko A, Głuszek S, Rydzewska G. Rapid fecal calprotectin test for prediction of mucosal inflammation in ulcerative colitis and Crohn disease: a prospective cohort study. Pol Arch Intern Med. 2017 May;127(5):312318 .
19. Ricanek P, Brackmann S, Perminow G, Lyckander LG, Sponheim J, Holme O, et al. Evaluation of disease activity in IBD at the time of diagnosis by the use of clinical, biochemical, and fecal markers. Scand J Gastroenterol. 2011 Sep;46(9):1081-91. DOI: 10.3109/00365521.2011.584897

20. Lobatón T, Bessissow T, De Hertogh G, Lemmens B, Maedler C, Van Assche G, et al. The Modified Mayo Endoscopic Score (MMES): A New Index for the Assessment of Extension and Severity of Endoscopic Activity in Ulcerative Colitis Patients. J Crohns Colitis. 2015 Oct;9(10):846-52. DOI: 10.1093/ecco-jcc/jjv111

21. Sandborn WJ, van Assche G, Reinisch W, Colombel JF, D'Haens G, Wolf DC, et al. Adalimumab induces and maintains clinical remission in patients with moderate-to-severe ulcerative colitis. Gastroenterology. 2012 Feb;142(2):257-65.e1-3. doi: 10.1053/j.gastro. 2011. 10.032. Epub 2011 Nov 4.

22. Schoepfer AM, Beglinger C, Straumann A, Trummler M, Renzulli P, Seibold F. Ulcerative colitis: correlation of the Rachmilewitz endoscopic activity index with fecal calprotectin, clinical activity, C-reactive protein, and blood leukocytes. Inflamm Bowel Dis. 2009 Dec;15(12):1851-8. DOI: 10.1002/ibd.20986

23. Schoepfer AM, Beglinger C, Straumann A, Safroneeva E, Romero Y, Armstrong D, et al. Fecal calprotectin more accurately reflects endoscopic activity of ulcerative colitis than the Lichtiger Index, C-reactive protein, platelets, hemoglobin, and blood leukocytes. Inflamm Bowel Dis. 2013 Feb;19(2):332-41. DOI: 10.1097/MIB.0b013e3182810066

24. D'Haens G, Ferrante M, Vermeire S, Baert F, Noman M, Moortgat L, et al. Fecal calprotectin is a surrogate marker for endoscopic lesions in inflammatory bowel disease. Inflamm Bowel Dis. 2012 Dec;18(12):221824. DOI: $10.1002 /$ ibd. 22917

25. National Institute for Health and Care Excellence. Fecal calprotectin diagnostic tests for inflammatory diseases of the bowel. Diagnostics guidance. 2013 Oct:1-56. 